\title{
Application of the Technique Stereotactic Body Radiotherapy (SBRT) in Pulmonary Metastases of Sarcomatoso Origin, Review of Highlighted Literature
}

\author{
Mariana Perez De Antueno ${ }^{1 *}$ and Ana Sebio García ${ }^{2}$ \\ ${ }^{1}$ Medical Radiotherapist, Argentina \\ ${ }^{2}$ Medical Oncologist, Barcelona, Argentina
}

*Corresponding author: Mariana Perez De Antueno, Medical Radiotherapist, Argentina.

Received Date: September 27, 2018

Published Date: December 14, 2018

\begin{abstract}
Stereotactic body radiotherapy (SBRT) is a radiotherapy technique that provides radiation from many different positions around the body. The rays are focused on the tumor, with this we achieve that the lesion receives a high dose of radiation and the tissues around it receive a low dose, thus reducing the risk of side effects. It is applied between 1 and 5 sessions, and therefore it is possible to reduce the treatment time compared with a conventional treatment. This radiation therapy is also called stereotactic ablative radiotherapy (SABR). The SBRT for the treatment of pulmonary sarcoma metastases can play an important role in the following circumstances:

a. Inoperable metastases at critical sites.

b. Recurrent metastases.

c. Inoperable patients due to limited lung function

d. And especially in metastasis of tumor types particularly resistant to the dose of radiotherapy used in previous SBRT techniques such as chordoma, chondrosarcoma, osteosarcoma, where, thanks to the development of SBRT, it is possible to deliver a higher biological dose. With this new technique, one can speak of providing excellent local control of pulmonary sarcoma metastases with an impact on survival in selected patients.
\end{abstract}

\section{Introduction}

The SBRT, body stereotactic radiotherapy, is a radiotherapy technique, which is characterized by the possibility of delivering radiation through multiple incidences, along an arch, thus achieving greater dose concentration in the tumor, limiting the dose in healthy tissues, and therefore minimizing late effects [1]. The $\alpha / \beta$ ratio is a measure of radiosensitivity, used in radiotherapy, to measure the response of tissues to radiation. It is understood that a low $\alpha / \beta$ ratio (from 0.1 to 5.4) in relation to the surrounding tissues, have a higher response at high doses, in little fractionation, while a high $\alpha / \beta$ ratio (from 6 to 14) have a greater response to long fractions, with low doses per day. Sarcomas are tumors with an $\alpha / \beta$ ratio between 0.5 to 5.4 according to histologists, therefore it is considered that they would have a better response to short fractionation, with high dose per day of radiation (1000 cGy / day for 5 days vs standard fractionation of 200 cGy / day for 20 days) [1-2].

This is the main reason that leads researchers to consider the possibility of performing this technique in patients with pulmonary sarcoma metastases, in which due to different reasons no other treatment option is allowed. The curative treatment of oligomethastasis with the SBRT radiotherapy technique is an active research area. Although there is no maximum consensus on the curability of the resection / treatment of pulmonary metastases, it is considered by many authors that the treatment of five or less pulmonary metastases of sarcomas would be performed with curative intent, while individuals with more of five metastases, the treatment would have a palliative purpose. This modality of radiotherapy, SBRT, is increasingly used in the treatment of sarcomas 
and among the current indications, can be listed: inoperable metastases in critical sites, recurrent metastases, 5 patients with limited lung function and especially patients with metastasis of tumors particularly resistant to conventional radiotherapy doses, as is the case of chordomas, chondrosarcomas and osteosarcomas, where the biological dose that can be delivered thanks to this technique may be greater than with other radiotherapy techniques. The pulmonary metastases of sarcoma are typically treated with surgical resection and / or chemotherapy. SBRT can be a new alternative to surgery, achieving high local control (LC) rates with limited toxicity. For this reason, in this work, a systematic review of the literary is carried out in order to assess this treatment modality.

The SBRT or ablative stereotactic radiotherapy (SABR), has its origins in the 90s, where some groups tried to administer stereotactic treatment to extracranial sites, imitating the technique of intracranial radiosurgery (SRS), already used since 1951, by Lars Leksell, who He challenged the radiobiological dogma of conventional intracranial fractionation. Hamilton and his colleagues, in 1995, tried to administer SRS to spinal tumors with rigid immobilization, screwing a framework to the spinous process of the vertebra. Unlike the intracranial SRS, where true rigid fixation is possible, the precision of the configuration was affected by the inherent movement. That is why every year, we try to improve these immobilization systems, so that they are increasingly simple in their use and more efficient. There are three main categories of movement control methods: motion damping (e.g. abdominal compression) (Figure 1), motion blockage, and movement tracking (e.g. active breath control with fiducial) (Figure 2). If none of the above methods is available, a four-dimensional tomography (4D) can be used to assess the individual mobility of the tumor and can be used to generate an ITV (internal target volume) for treatment planning (Figure 3). If the possibility of 4D CT is not available, a combination of three CT series obtained during free breathing, deep inspiration and deep expiration, can be used to generate the ITV [6].

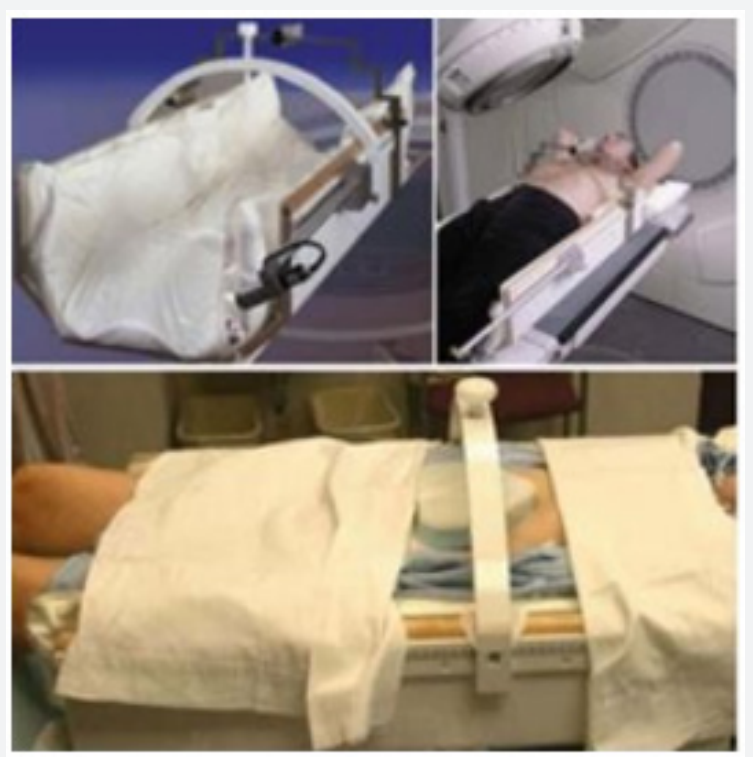

Figure 1: (Method 1) Motion Damping.

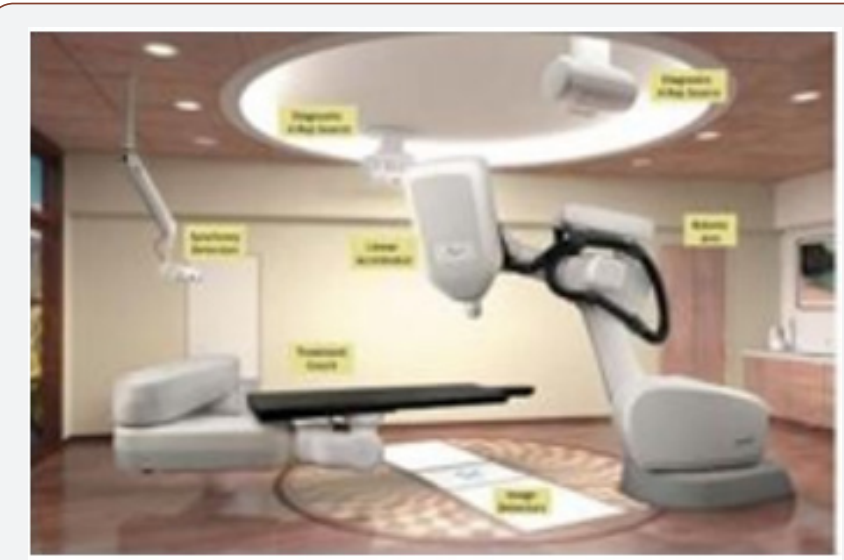

Figure 2: (Method 2) Motion Blockage, And Movement Tracking.

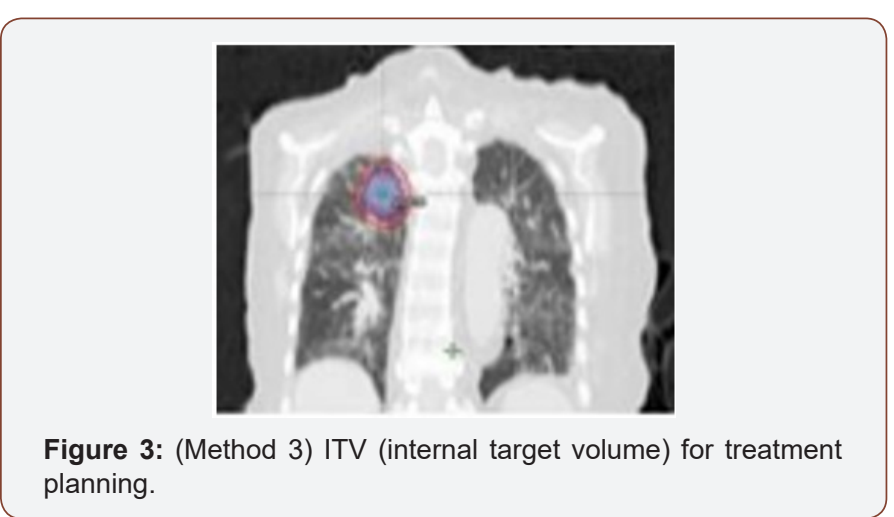

The SBRT or SABR technique, leads to the precise and focused delivery of a small number of radiation fractions, in the dose range ablative to extracranial targets. The advances in the technologies, facilitated the safe and effective delivery of SBRT in different organs. Clinical experience with the use of SBRT to treat various extracranial organ sites has increased exponentially in the last 5-10 years in North America, Europe, and Asia.

With this technique the radiation is usually delivered, through 1 to 5 fractions, trying to achieve that an ablative dose of radiation is administered to the tumor, while trying to preserve the healthy tissue of the environment or structures to avoid as much as possible a collateral damage [3]. When we talk about sarcomas, the most common primary site for metastatic disease is the lung, with bone deposits, intracranial, hepatic and other soft tissues less frequent. The average survival after the development of the secondary disease is 18 months, but with a fairly broad range. Important prognostic factors are, the number of metastases and the presence of bilaterality or not and the disease-free interval, since a longer interval predicts for greater survival after the development of metastasis. The best evidence base for the local treatment of pulmonary metastases, and where there is an extensive literature, is the surgical one, which mentions a 5-year survival of $25-50 \%$ for soft tissue sarcoma and $34-50 \%$ for osteosarcoma, in selected patients who underwent metastasectomy [4].

\section{Materials and Methods}

A systematic review of documents of scientific societies dedicated to sarcomas and radiotherapy was carried out, as well as reviews and scientific studies. As a search strategy, in the first place, 
a Google Scholar search was carried out of documents and protocols published by different societies and professional associations worldwide on radiotherapy in sarcomas. The search was conducted in both Spanish and English. Subsequently, a search was made of systematic reviews of the scientific literature, with no date limit, taking into account that they are from different cities, to make a global assessment. For the search of original studies, the Medline, PubMed, ScienceDirect and The Cochrane Library databases were consulted with no date limit, although the language was limited to English and Spanish. Bibliographic references were also analyzed in order to rescue other studies. Regarding the inclusion and exclusion criteria in the search of the gray literature, all types of documents contributed by the different societies and professional associations were included, which made recommendations on the treatment of pulmonary metastases of sarcomas with SBRT. The main exclusion criteria were articles that did not include reliable data on this treatment.

After the initial search, after discarding bibliography, information was obtained from 3 books and 11 articles. To proceed with the information, the abstracts were reviewed and, if necessary, the entire article was revised. Data analysis: from the information analyzed, authorship was extracted, the journal where it was published, year, purpose, source of information, type of study, results and conclusions.

\section{Result}

Stereotactic radiotherapy in cases of pulmonary sarcoma metastasis has been reported by several authors and has been associated with a local control rate of $90 \%$ at 2 years and $85 \%$ at 3 years with a delivered dose of 50-60. Gy in 4-6 fractions and survival comparable to surgery that is 75 and $63 \%$ at 2 and 3 years respectively [4]. In the year 2012, Dhakal S, et al. [5] to the published an article called "Stereotactic body radiotherapy for pulmonary metastases from soft tissue sarcomas", in this reading expresses the excellent local control of the lesion and better patient survival5. In this work, the authors describe the results since 2001 of pulmonary metastases of sarcomas treated with SBRT. A total of 52 cases were retrospectively reviewed. The majority of patients received multimodal treatment that included surgery, chemotherapy and or radiation. Leiomyosarcoma (23\%), malignant fibrous histiocytoma (19\%) and synovial sarcoma (15\%) were the most common histologies. The median number of lesions treated was 4 (1-16) per patient and 3 (1-6) sessions. The preferred dose and the fractionation were $50 \mathrm{~Gy}$ in fractions of $5 \mathrm{~Gy}$. The LC (local control) at three years was $82 \%$. The median overall survival was $2.1(0.8-11.5)$ years for patients treated with SBRT and 0.6 (0.1-7.8) years for those who never received SBRT ( $p=0.002)$. In conclusion, this study group reports that the SBRT radiotherapy technique provides excellent local control of lung metastases and an increase in survival. SBRT should be considered, according to the authors, for all patients with MP of STS, particularly those who are not candidates for surgery [5].

In that same year, C. Stragliotto et. to the. published in the journal Medical Oncology, an article where they retrospectively reviewed the results of SBRT in 46 patients, with a total of 136 metastases of irradiated primary sarcoma. The objective was to evaluate the overall response rate and the side effects of SBRT in metastatic sarcoma. Patients were treated between 1994 and 2005. With respect to data on fractionation sensitivity for sarcomas, 10 are very rare. However, it is reported that some types of sarcoma have a very low $\alpha / \beta$ ratio, for example, they reported an $\alpha / \beta$ value of approximately 1 for osteosarcoma and an $\alpha / \beta$ ratio of 0.4 for liposarcoma. Due to the complexity in fractionation sensitivity for different types of sarcoma, the $\alpha / \beta$ ratio generally used is 3 for all tumors in this study. The prescribed doses ranged from 4 to 20 Gy per fraction in 1-5 fractions, with total doses of 10-48 Gy. The metastases treated were located mainly in the lungs. A total of 136 metastases were treated (114 per patient). The disease control rate (complete response, partial response and stable disease) was $88 \%$ (119/135). The median follow-up was 21.8 months (range 2.7-112.8 months). Thirteen patients (31\%) were long-term survivors (> 36 months) and 5 patients are still alive after the last follow-up. Two cases of severe non-lethal side effects were observed, one patient had a colon perforation and another patient had a contracture of the hip region. Radiation therapy administered with the conventional technique is sometimes used for palliative treatment, but doses administered per fraction are often considered too low to achieve adequate local control, and the results are disappointing. The radiotherapy administered with SBRT technique, delivers hypo fractioned doses in a short period of time, this can provide curable doses and the possibility of a high local control. In conclusion they report that SBRT is a safe, convenient and effective non-invasive treatment with high local control for patients with metastatic sarcoma [6].

In the year 2013, Mehta N, et al. [7] publish an article entitled "Safety and Efficacy of Stereotactic Body Radiation Therapy in the Treatment of High-Grade Sarcoma Pulmonary Metastases". In this work, SBRT is described as an alternative treatment in pulmonary metastases of sarcomatous origin. They performed this treatment in 16 patients with pulmonary metastases of highgrade sarcoma. All 16 patients received chemotherapy and a subset (38\%) also underwent previous pulmonary metastasectomy. A total of 25 lesions were treated and evaluated for this analysis. The most common histologies were leiomyosarcoma (28\%), synovial sarcoma (20\%) and osteosarcoma (16\%). The average dose of SBRT prescription was 54 Gy (36-54) in 3-4 fractions. At 43 months, local control was 94\%. No patient 11 experienced G2-4 radiation pneumonitis and no patient experienced radiation esophagitis. In their conclusion, they state that they suggest performing the SBRT technique in pulmonary sarcoma metastases since this modality provides excellent local control and minimal toxicity. In 2015, Navarria P, et al. [8] to the published in the European Journal of Cancer, an article on "Stereotactic body radiotherapy for pulmonary metastases from soft tissue sarcomas". They reviewed the treatment of 51 injuries, of 21 patients in total. All the patients were in good functional condition, but they were not suitable for surgical resection, with primary tumor controlled and the number of lung metastases was $\leqslant 4$. In a scheme of adaptation to risk, the dose prescription was: $30 \mathrm{~Gy} / 1 \mathrm{fr}, 60 \mathrm{~Gy} / 3 \mathrm{fr}, 60 \mathrm{~Gy} / 8 \mathrm{fr}$ and $48 \mathrm{~Gy} / 4$ fr. The treatments were performed with volumetric archmodulated therapy. The clinical result was evaluated by thoracic 
and abdominal computed tomography (CT) before the SBRT and every 3 months. Toxicity was assessed with version 4.0 of Common Terminology Criteria for Adverse Events (CTCAE). Among the cases evaluated, leiomyosarcoma (36\%) and synovial sarcoma (25\%) were the most frequent histologies. Five patients (18\%) initially presented pulmonary metastases, while $23(82 \%)$ developed them at a median of 51 months (range 11-311 months) from the initial diagnosis. The median follow-up time from the initial diagnosis was 65 months (5-139 months) and from the SBRT was 21 months (2-80 months). No severe toxicity was recorded (grades III-IV) and no patient required hospitalization. The actuarial local control rate at 5 years was $96 \%$. Overall survival at 2 and 5 years was $96.2 \%$ and $60.5 \%$, respectively. At the last follow-up, 15 patients (54\%) were alive and the rest of the patients died by distance progression. In conclusion, the authors highlight that SBRT provides excellent local control of pulmonary metastasis of soft tissue sarcoma (STS) and can improve Survival in selected patients. SBRT should be considered for all patients with pulmonary metastases and should be evaluated in a multidisciplinary team.

Also, in 2015 Frakulli R, et al. [9] published "Stereotactic radiotherapy in the treatment of lung metastases of bone and sarcomas of 12 soft tissues", where its objective was to evaluate local control and toxicity in a group of patients treated with SBRT for pulmonary metastases of soft tissue sarcomas and bone. The dose delivered varied from 30 to 60 Gy in 3-8 fractions. Acute and late toxicity was rated according to the Common terminology criteria for adverse events version 4.0. In this study a total of 24 patients with 68 lung metastases from sarcomas were treated with SBRT. The median follow-up after performing the SBRT was 17 months (range = 1151 months). Local control of the twoyear actuarial lesion and overall survival were $85.9 \%$ and $66.4 \%$, respectively. No acute or late G3 or G4 toxicities were observed. The authors concluded that SBRT is a safe and effective treatment for the treatment of pulmonary sarcoma metastases and could be used as an alternative option in patients not suitable for surgery. In July of the year 2016, Baumann B, et al. [10] they try to demonstrate "The efficacy and safety of stereotactic body radiotherapy for the treatment of pulmonary sarcoma metastases" and report it as a potential alternative to resection. In this publication, they describe having performed SBRT in 30 patients with pulmonary sarcoma metastases. The treatment consisted of an average dose of $50 \mathrm{~Gy}$ in 4-5 fractions with CyberKnife or linear accelerator. $77 \%$ of patients received previous chemotherapy, $70 \%$ had $1-3$ previous lung resections and $26 \%$ had previous chest radiotherapy. The median size of the lesion was $2.4 \mathrm{~cm}$ (range $0.5-8.1 \mathrm{~cm}$ ). The authors concluded that the SBRT technique is well tolerated by patients, with excellent local control since this was $94 \%$ and $86 \%$, and OS was $76 \%$ and $43 \%$ at 12 and 24 months. Overall survival and local control did not differ according to SBRT technique, fractionation regimen, lesion location, histology or size, (P 0.05) in all locations and sizes of tumors, the authors suggest that SBRT should be considered and that prospective studies are justified.

Also, in 2016 Sapir E [11] published in the journal, Radiation Oncology, an article called "Surgical resection or stereotactic therapy of body radiation for patients with sarcoma with pulmonary metastases", whose purpose was the aggressive management of sarcometic oligometastases, which may result in long-term survival and a delay in the administration of chemotherapy. In this study, 78 patients with 127 lung lesions were treated with surgical resection and 26 patients with 47 lung metastases underwent ablation with SBRT. The median tumor size was $1.5 \mathrm{~cm}(0.1-12.8)$ and $1.4 \mathrm{~cm}$ (0.4-4.8), respectively, for lung metastases administered surgically and with SBRT. On average, the patients in the SBRT group were older (age $60 \mathrm{v} 52, \mathrm{P}=0.03$ ), had received more previous systemic therapies (1.98 vs. $1.29, \mathrm{P}=0.01$ ), and had a greater presence of active extrathoracic disease at the start of treatment. (59.6\% vs. $29.9 \%, \mathrm{P}<0.01$ ). Local control was not different between groups when adjusted for size, other sites of active disease, age and functional status ( $\mathrm{HR}=1.47,95 \%$ CI $0.20-10.9, \mathrm{P}=0.71)$. The 1-year and 2-year local progression-free rate for all lung lesions resected versus SBRT were $96.8 \%$ and $96.8 \%$ versus $97.4 \%$ and 97.4\%. Overall survival (OS) at 1 and 2 years was $92.6 \%$ and $62.2 \%$ for surgery patients and $88.1 \%$ and $57.9 \%$ for SBRT, with no differences between the groups (HR $=0.71,95 \% \mathrm{CI}=0.39$ $1.29, \mathrm{P}=0.27$ ) The progression-free survival (PFS) at 1 and 2 years was $33.4 \%$ and $15.7 \%$ for patients undergoing surgery, $42.3 \%$ and $21.6 \%$ for patients with SBRT, without significant differences between the groups $(\mathrm{HR}=1.21,95 \% \mathrm{CI}=0.87-1.68, \mathrm{P}=0.25)$. Grade 3+ adverse events were observed in five patients treated with surgery versus none in patients with SBRT. Conclusion of this series was that SBRT provides local control, SLP and similar SG compared to surgical resection of pulmonary sarcoma metastases, and therefore can serve as a safe alternative to surgical resection. However, the authors note that a randomized prospective study is still required to determine if there is a comparable long-term survival with SBRT versus surgery for the treatment of sarcoma lung metastases.

In that same edition of the journal in October 2016, Lindsay A [12] of the University of Florida, publishes together with his collaborators an article called "Efficacy of the SBRT in the treatment of metastasis of sarcoma in the lung: experience in a center of tertiary reference ". The purpose of this work is to evaluate the effectiveness and safety of SBRT, for the definitive ablative treatment of pulmonary metastases of primary sarcoma, in patients for whom surgical resection is technically or medically at high risk. For this, SBRT was used in 117 lung metastases of soft tissue sarcoma of 44 patients for whom resection was not practical due to the surgical risks in excess. The predominant sarcoma histology was undifferentiated pleomorphic sarcoma / malignant fibrous histiocytoma (22 patients). The median number of metastases treated per patient was 2 (range, 1-7). Seventeen patients had previous thoracic surgery for metastatic disease and 23 patients received previous chemotherapy treatment. The majority (84\%) of the metastases were treated with a total dose of 50 Gy. Daily dose fractions of 10 Gy were administered in $68 \%$ of the treatments, and $11 \%$ were treated with daily dose fractions of $5 \mathrm{~Gy}$. In patients with multiple metastatic sites, all lesions were treated at the same time with SBRT treatment plans. The patients were followed with serial CT scan every 3 months. The mean follow-up of this series was 19.3 months (range, 1,698.6 months). The overall survival rate was $82 \%$ at 2 years and $50 \%$ at 5 years. Only 4 patients experienced 
disease progression in metastatic sites treated with SBRT. Of the 117 metastatic nodules treated, only 6 sarcoma metastases showed treatment failure due to volumetric progression. This represents a 95\% overall control rate for sarcoma metastases treated with SBRT. Overall survival did not improve in patients who received treatment with chemotherapy (median survival was 52.4 months with chemotherapy versus 74.3 months without; $P$ value $=0.161$ ) Side effects of SBRT were predominantly grade 1-2 transient pneumonitis $(\mathrm{n}=6)$, cough $(\mathrm{n}=3)$, dermatitis $(\mathrm{n}=1)$ and dyspnea $(n=1)$. Late effects were limited to 1 patient who experienced a rib fracture and chronic pain. In conclusion, the authors remarked that SBRT is a highly effective and safe treatment for the ablation of pulmonary metastases from primary sarcoma. In addition, they conclude that an additional clinical study is justified to evaluate the specific factors of the patient and the disease in the definition of the role optimal of SBRT in relation to surgery and chemotherapy for the treatment of oligometastatic sarcoma.

\section{Conclusion and Future Perspectives}

The curative treatment of oligomethastasis with the SBRT radiotherapy technique is an active research area.

To date, retrospective studies have shown that the use of SBRT for oligorecurrence achieved acceptable tumor control and the additional SBRT was also effective for selected patients with a second oligorecurrence after the use of SBRT in a previous metastatic lesion [13]. Although the selection of patients with pulmonary metastasis of sarcomas is currently complex for the realization of SBRT, new prospective studies are expected in the near future to demonstrate that this technique is comparable to surgical resection, in local control and survival, not to mention the importance of being a non-invasive technique and with good tolerance and acceptance by the patient. That is why it is important that all the multidisciplinary team that works and dedicates itself to the study and treatment of patients with sarcomas, is aware of the existence of this modality of radiotherapy, which can be indicated as part of the treatment protocol and that day to day proves to be more promising. Example of active research in this field, is a pilot study opened in April 2017 in which the SBRT and adaptive radiotherapy (TAR, radiotherapy technique, which adapts the treatment to tumor volume in each fraction) for pulmonary metastasis is evaluated. of soft tissue sarcomas. This is a non-randomized, single arm study that evaluates SBRT with TAR for the treatment of unresectable lung metastases from a primary soft tissue sarcoma. Each metastatic lesion will receive 3 to 5 fractions. The primary objective is safety and toxicity and secondary outcomes include local / regional control, quality of life and overall survival. Currently there is also a phase II study of observational research, cohort modality, to evaluate the viability and safety of SBRT for pulmonary metastases of soft tissue sarcoma. This study was started in April 2015 and is expected to be completed in 2020. The primary objective is the local control rate of the treated lesions and as secondary objectives the overall survival and disease-free survival of the treated patients will be evaluated [14].

In conclusion, the treatment of pulmonary metastases of sarcoma with SBRT seems to be a good therapeutic alternative in selected patients with a good toxicity profile. However, randomized studies are necessary to confirm the available retrospective data and obtain more information on the adequate selection of patients who can benefit from this treatment.

\section{Acknowledgement}

We would like to thank Dr. Ana Sebio García, Medical Oncologist, Barcelona, and Santiago Girola, Medical Physician of Argentina, for the support and assistance in this study.

\section{Conflict of Interest}

None of the authors had any financial or personal relationship with persons or organizations that could unduly influence this work.

\section{References}

1. Halperin EC, Brady LW, Perez CA (2008) Perez \& Brady's Principles and Practice of Radiation Oncology. Lippincott Williams \& Wilkins Philadelphia, PA, USA.

2. Viacheslav Soyfer, Benjamin W Corn, Yehuda Kollender, Haim Tempelhoff, Isaac Meller, et al. (2010) Radiation Therapy for Palliation of Sarcoma Metastases: A Unique and Uniform Hypofractionation Experience. Hindawi/journals/sarcomas. [Internet] Volume 2010: 4.

3. Stereotactic Body Radiation Therapy. In: Lo SS, Teh BS, Lu JJ, Schefter TE (Eds.), Springer Heidelberg. (2012 edn), New York, USA.

4. Stereotactic Body Radiotherapy: A Practical Guide. In: Gaya, Andrew, Mahadevan, Anand (Eds.), (2015 th $e d n)$, Springer-Verlag, New York, USA.

5. Dhakal S, Corbin KS, Milano MT, Philip A, Sahasrabudhe D, et al. (2012) Stereotactic body radiotherapy for pulmonary metastases from softtissue sarcomas: excellent local lesion control and improved patient survival. Int J Radiat Oncol Biol Phys 82(2): 940-945.

6. Stragliotto CL, Karlsson K, Lax I, Rutkowska E, Bergh J, et al. (2012) A retrospective study of SBRT of metastases in patients with primary sarcoma. Med Oncol 29(5): 3431-3439.

7. Mehta N, Selch M, Wang P, Federman N, Lee JM, et al. (2013) Safety and Efficacy of Stereotactic Body Radiation Therapy in the Treatment of Pulmonary Metastases from High Grade Sarcoma. Journal List Sarcoma 2013: 360214.

8. Navarria P, Ascolese A, Luca Cozzi L, Tomatis S, Giuseppe Roberto D’Agostino, et al. (2015) Stereotactic body radiation therapy for lung metastases from soft tissue sarcoma. European Journal of Cancer 51(5): 668-674.

9. Rezarta Frakulli R, Salvi F, Balestrini D, Parisi A, Marcella P, et al. (2015) Stereotactic Radiotherapy in the Treatment of Lung Metastases from Bone and Soft-tissue Sarcomas. Anticancer Research, International Journal of Cancer Research and Treatment 35(10): 5581-5586.

10. Baumann BC, Nagda SN, Kolker JD, Levin WP, Weber KL, et al. (2016) Efficacy and safety of stereotactic body radiation therapy for the treatment of pulmonary metastases from sarcoma: A potential alternative to resection. J Surg Oncol 114(1): 65-9.

11. Sapir E, Tao Y, Lin J, Kollar L, Schipper M, et al. (2016) Surgical Resection or Stereotactic Body Radiation Therapy for Sarcoma Patients with Pulmonary Metastases. Radiation Oncology, ASTRO 96(2): S26.

12. Lindsay A, Haupt E, Chan C, Spiguel A, Scarborough MT, et al. (2016) Effectiveness of Stereotactic Body Radiation Therapy in Treatment of Sarcoma Metastasis to the Lung: Experience at a Tertiary Referral Center. Radiation Oncology, ASTRO 96(2): S67.

13. Aoki M, Hatayaman Y, Kawaguchi H, Hirose K, Sato M, et al. (2016) Stereotactic body radiotherapy for lung metastases as oligo-recurrence: a single institutional study. J Radiat Res 57(1): 55-61.

14. Tedeschi M (2015) SBRT Treatment for Lung Metastasis from Soft Tissue Sarcoma. Istituto Clinico Humanitas. 